\title{
PRIMEIRO REGISTRO DO MORCEGO MIMON CRENULATUM (PHYLLOSTOMIDAE) NO PANTANAL, SUDOESTE DO BRASIL
}

\author{
George Camargo $^{1}$ \& Erich Fischer ${ }^{2}$
}

Biota Neotropica v5(n1) - http://www.biotaneotropica.org.br/v5n1/pt/abstract?short-communication+BN00705012005

\author{
Recebido: 23/08/2004 \\ Revisado: $11 / 11 / 2004$ \\ Publicado: 01/01/2005
}

\begin{abstract}
${ }^{1}$ Programa de PG em Ecologia e Conservação (http://www.dbi.ufms.br/mstecopan/ecologia.htm), Centro de Ciências Biológicas e da Saúde, Universidade Federal de Mato Grosso do Sul, 79070-900 Campo Grande, Brasil ${ }^{2}$ Departamento de Biologia, Universidade Federal de Mato Grosso do Sul (http://www.ufms.br), CP 549, 79070-900 Campo Grande, Brasil (autor para correspondência; efischer@nin.ufms.br)
\end{abstract}

\begin{abstract}
Species of Mimon Gray, 1847, are known to occur from Mexico to central Bolivia and southeastern Brazil, ranging from $22^{\circ} \mathrm{N}$ to $25^{\circ} \mathrm{S}$ of latitude. In latitudes between 14 and $25^{\circ} \mathrm{S}$, approximately, the known occurrence of Mimon is restricted to east Brazil, in the Atlantic rainforest. Only in latitudes lower than $14^{\circ} \mathrm{S}$ the species of Mimon additionally occurs in central and western South America, in the Amazon, Cerrado and Caatinga. Mimon crenulatum (E. Geoffroy, 1810) is locally uncommon but widespread over the range of the genus distribution. We report here the occurrence of $M$. crenulatum at ca. $20^{\circ} \mathrm{S}$ of latitude in the Pantanal wetlands, western Brazil. It is probably the first record of the genus Mimon in this ecosystem, and it increases the geographic range of M. crenulatum distribution to western Brazil in latitudes greater than $14^{\circ} \mathrm{S}$.
\end{abstract}

Key words: bat distribution, geographic range, Mimon, Pantanal, Phyllostomidae.

\section{Resumo}

Espécies do gênero Mimon Gray, 1847, ocorrem desde o México até a Bolívia central e sudeste do Brasil, entre as latitudes $22^{\circ} \mathrm{N}$ e $25^{\circ} \mathrm{S}$. Aproximadamente entre 14 e $25^{\circ} \mathrm{S}$ de latitude, a ocorrência conhecida de Mimon é restrita ao leste brasileiro, na Mata Atlântica. Apenas em latitudes menores que $14^{\circ} \mathrm{S}$ as espécies de Mimon adicionalmente ocorrem na região central e oeste da América do Sul, na Amazônia, Cerrado e Caatinga. Mimon crenulatum (E. Geoffroy, 1810) é localmente rara porém ocorre amplamente dentro dos limites de distribuição do gênero. Reportamos aqui a ocorrência de Mimon crenulatum a ca. $20^{\circ}$ de latitude sul na planície inundável do Pantanal, oeste do Brasil. Provavelmente este é o primeiro registro do gênero Mimon neste ecossistema, ampliando a distribuição geográfica de M. crenulatum para o oeste brasileiro em latitudes maiores que $14^{\circ} \mathrm{S}$.

Palavras-chave: distribuição geográfica, morcegos, Mimon, Pantanal, Phyllostomidae. 


\section{INTRODUÇÃO}

Espécies do gênero Mimon Gray, 1847, ocorrem desde o sul do México até a Bolívia central e sudeste do Brasil (Emmons \& Feer 1990). Existem duas ou três espécies no gênero: Mimon crenulatum, M. bennettii e M. cozumelae, que alguns autores consideram como sinônimo júnior de $M$. bennettii (Jones et al. 1988, Genoways et al. 1981, Ortega \& Arita 1997, Koopman 1993). Mimon crenulatum(E. Geoffroy, 1810) é amplamente distribuída na América Central e norte da América do Sul, porém apresenta distribuição restrita ao leste da América do Sul entre 14 e $25^{\circ} \mathrm{S}$ de latitude (Fernandez et al. 1988, Medellín 1983, Ochoa et al. 1993). No Brasil, $M$. crenulatum foi registrada em diferentes tipos de vegetação como Floresta Amazônica, Cerrado, Caatinga e Mata Atlântica, não tendo sido encontrado registro de ocorrência no Pantanal (q.v. revisão em Marinho-Filho \& Sazima 1998). Reportamos aqui a ocorrência de M. crenulatum no Pantanal, região de Nhecolândia, Mato Grosso do Sul.

\section{MATERIAL E MÉTODOS}

O Pantanal compreende extensa planície inundável situada aproximadamente entre 14 e $22^{\circ} \mathrm{S}$ de latitude e entre 53 e $66^{\circ} \mathrm{O}$ de longitude. $\mathrm{O}$ clima da região é tropical quente, com inverno seco (Aw de Köppen). A pluviosidade média anual é de aproximadamente $1100 \mathrm{~mm}$, com chuvas concentradas entre novembro e março. A paisagem é determinada, principalmente, pelos pulsos anuais de cheia e vazante (Adámoli 1986). A vegetação é composta por campos de gramíneas, formações florestais e vegetação aquática (Prance \& Schaller 1982). A região do Pantanal em Nhecolândia é caracterizada principalmente pela presença de lagoas, denominadas "baías” ou "salinas”. As salinas são desconectadas do lençol freático e de cursos d’água, apresentando águas alcalinas com bicarbonato de cálcio. As baías e salinas são circundadas por extensos cordões de floresta, denominados “cordilheiras”. Outros hábitats florestais na região da Nhecolândia são as matas ciliares e os “capões”, que compreendem ilhas (0,2 a 4 ha) de floresta semidecídua circundadas por campos inundáveis (Damasceno et al. 1999, Araujo 2003) .

Espécimes de morcegos foram capturados com auxílio de redes de neblina, entre setembro de 2002 e dezembro de 2003, na fazenda Rio Negro, Pantanal da Nhecolândia, município de Aquidauana, Mato Grosso do Sul. Foram 15 expedições de campo, com 38 noites de amostragem, alternadas entre cordilheiras, matas ciliares e capões. A cada noite foram abertas dez redes de 12 x 2,6 m, sendo seis no interior e quatro na borda das formações florestais. Nas bordas de cordilheiras, em cada noite foram armadas duas redes próximas a salinas e duas redes próximas a baías. As redes foram abertas por volta de $1800 \mathrm{~h}$ e fechadas a $0100 \mathrm{~h}$. Todos os morcegos capturados foram identificados no campo e no mínimo um exemplar de cada espécie foi coletado para confirmação da identificação e inclusão nas coleções da UFMS ou do Laboratório de Quirópteros da Universidade para o Desenvolvimento do Estado e da Região do Pantanal - Uniderp, em Mato Grosso do Sul.

\section{RESULTADOS}

Foram registrados dois indivíduos de Mimon crenulatum dentre 438 morcegos capturados, pertencentes a 18 espécies. Considerando apenas as noites de amostragens em florestas de cordilheira, foram capturados 126 indivíduos de 10 espécies. Ambos indivíduos de $M$. crenulatum foram capturados em horário (0040 e 0045 h) e habitat (cordilheira) semelhantes, mas em locais e datas diferentes. O primeiro indivíduo (Figura 1) foi capturado em 29 de janeiro de 2003, a 19³0'16”' S e 56²'40”' O; e o segundo indivíduo em 30 de abril de 2003, a 19³3’03”' S e 56¹2’43”' O. Outra semelhança foi que ambos indivíduos de M. crenulatum foram capturados em redes marginais a salinas, na borda das cordilheiras. O primeiro indivíduo foi solto na mesma noite da captura e o segundo foi coletado e depositado na coleção zoológica da Universidade Federal de Mato Grosso do Sul (no. RNBS379). Este morcego era macho adulto pesando $15 \mathrm{~g}$ e apresentando as seguintes características: $48 \mathrm{~mm}$ de comprimento de antebraço, 22,8 mm de comprimento do crânio, 8,8 mm de largura da caixa craniana, 20,3 mm comprimento condilobasal, 4,5 mm constrição pós orbital, 12,5 mm largura do zigomático, 7,9 mm de comprimento maxilar, 8,8 mm comprimento mandibular e 9,0 mm entre molares superiores.

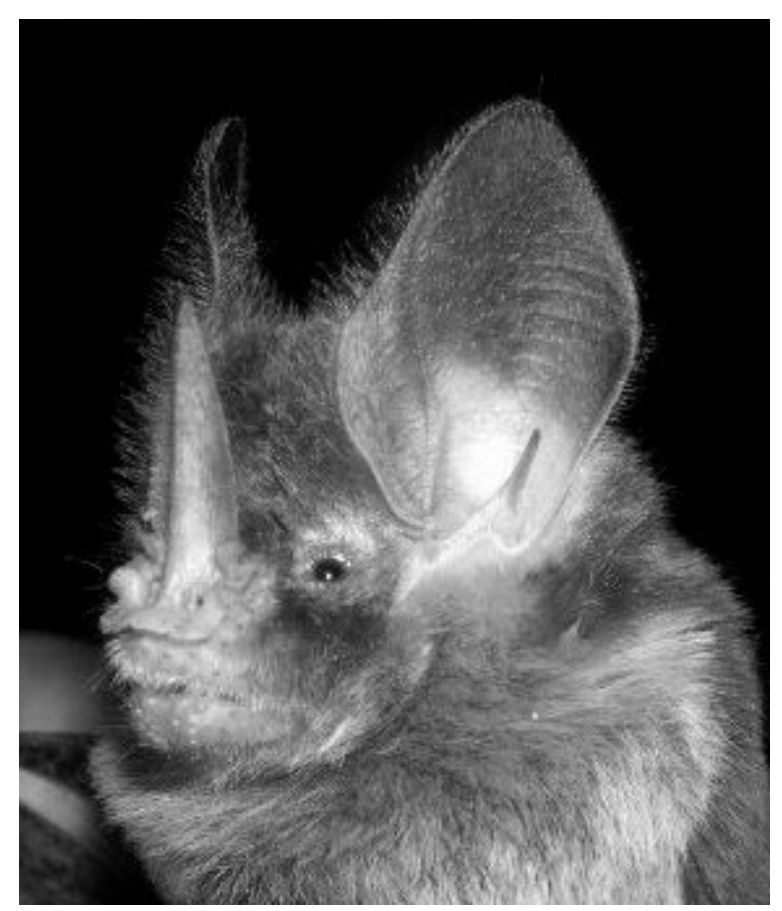

Figura 1. Espécime de Mimon crenulatum (Phyllostomidae) capturado no Pantanal da Nhecolândia, Mato Grosso do Sul. 


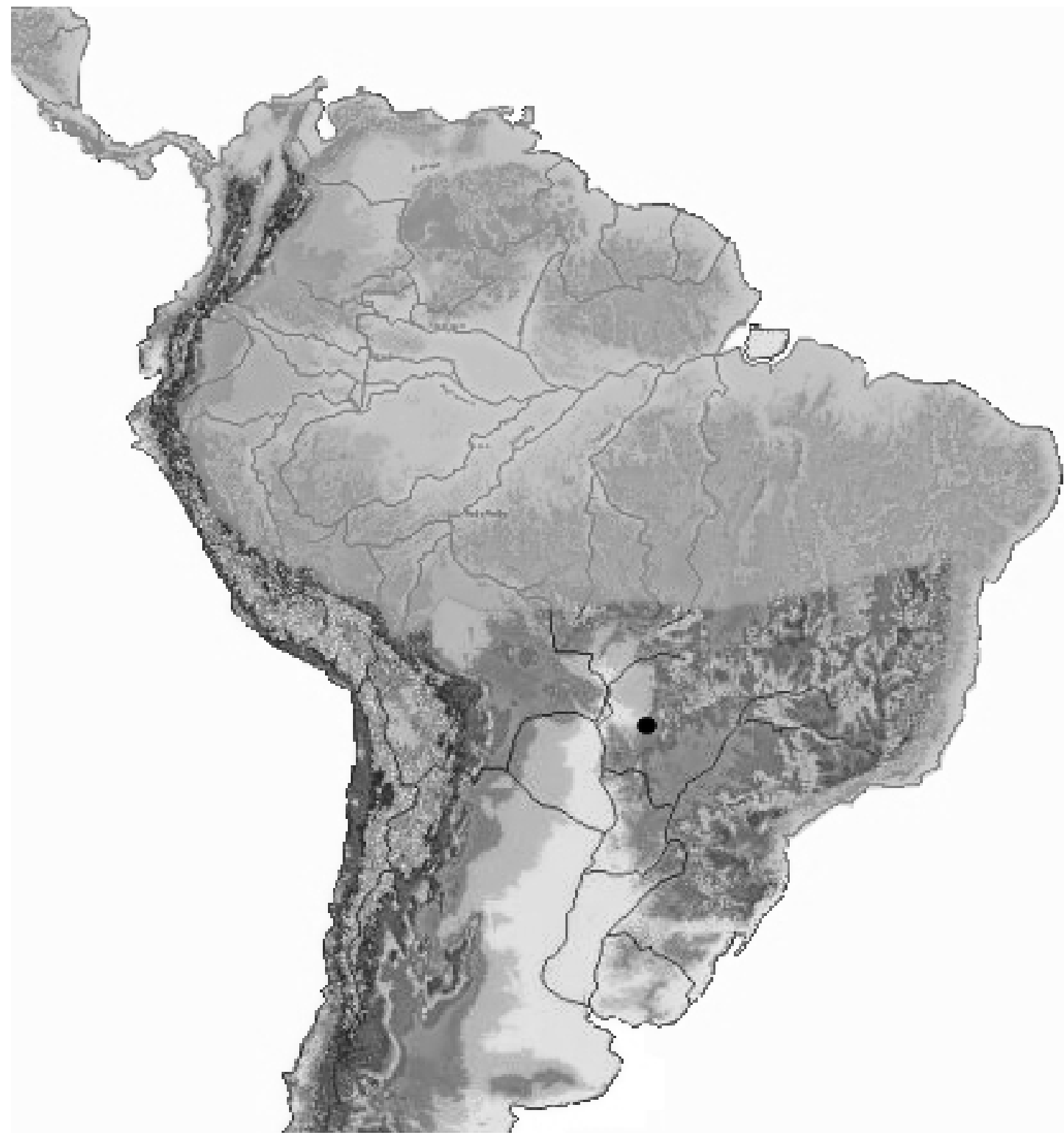

Figura 2. Mapa da distribuição conhecida para Mimon crenulatum (Phyllostomidae) na América do Sul (região em vermelho) (cf. Emmons \& Feer 1990); e região do Pantanal onde foi feito novo registro desta espécie (ponto negro)(presente estudo).

\section{DISCUSSÃO}

A baixa proporção (2/438) de capturas registrada para $M$. crenulatum indica que esta espécie é rara na região da fazenda Rio Negro, assim como reportado para esta espécie em outras regiões (Emmons \& Feer 1990). Mesmo considerando apenas morcegos capturados em cordilheiras, ainda assim M. crenulatum ocorreu em baixa proporção (2/ 126). As cordilheiras, muitas vezes, apresentam vegetação característica de Cerrado (como cerradão), tipo de vegetação onde a ocorrência de $M$. crenulatum já foi registrada (Pedro et al. 1994, Marinho-Filho \& Sazima 1998). Em outra parte do sul do Pantanal , região do rio Miranda, onde não ocorrem florestas de cordilheira, estudo anterior resultou em 639 morcegos coletados durante dois anos, não sendo registrado M. crenulatum (Camargo 2003). Portanto, a possibilidade de $M$. crenulatum estar associada às florestas de cordilheira no Pantanal parece ser questão interessante para ser investigada.

O registro de $M$. crenulatum em cordilheiras no Pantanal em Nhecolândia (presente estudo) amplia os limites de distribuição da espécie e do gênero de 14 para $20^{\circ}$ de latitude sul aproximadamente na região central da América do Sul; e amplia a distribuição de 50 para $56^{\circ}$ de longitude oeste em latitudes superiores a $14^{\circ} \mathrm{S}$ (Figura 2). Além de ampliar os limites de distribuição geográfica, este é provavelmente o primeiro registro nos domínios do Pantanal (q.v. Marinho-Filho \& Sazima 1998), ampliando a riqueza de 
ambientes de ocorrência da espécie e do gênero. A ocorrência de $M$. crenulatum em cordilheiras no Pantanal da Nhecolândia indica que esta espécie poderia também ocorrer em toda região sul do cerrado, bem como ao norte do Pantanal. A falta de inventários de morcegos em diferentes partes do Pantanal e na porção sul do Cerrado, combinada ao fato da espécie ser localmente rara (Emmons \& Feer 1990, presente estudo), poderia explicar a ausência de registros de $M$. crenulatum nestas regiões.

O macho de $M$. crenulatum coletado no local de estudo apresentou algumas medidas pouco abaixo da média registrada para machos adultos desta espécie no Cerrado (Pedro et al. 1994), como comprimento de antebraço, comprimento do crânio, comprimento condilobasal, comprimento maxilar e mandibular. Outras medidas foram semelhantes à média obtida por Pedro et al. (1994), como comprimento da constrição pós-orbital e largura da caixa craniana; porém a largura do zigomático do espécime coletado no Pantanal foi maior que a média registrada no Cerrado. São necessários mais registros de indivíduos no Pantanal para verificar se há características morfológicas diferentes para populações de $M$. crenulatum nesta região.

\section{AGRADECIMENTOS}

Ao Alan Fecchio, Andréa Araujo, Eurico Sczesny Moraes, Natália C. Campos e voluntários do Earthwatch Institute (EWI) pela valiosa ajuda no campo e no laboratório; aos funcionários da fazenda Rio Negro e ao staff da Conservação Internacional do Brasil (CI) e EWI pelo apoio logístico; ao Laboratório de Quirópteros da Universidade para o Desenvolvimento do Estado e da Região do Pantanal - Uniderp pelo apoio para identificação dos morcegos; ao CNPq, CI, EWI e Fundect pelo financiamento. Dedicamos este trabalho à memória do professor Valdir Antônio Taddei.

\section{REFERÊNCIAS BIBLIOGRÁFICAS}

ADÁMOLI, J.A. 1986. A dinâmica de inundações no Pantanal. In Anais do I Simpósio sobre Recursos Naturais e Socioeconômicos do Pantanal. CPAP Embrapa, Corumbá, p. 51-62.

ARAUJO, A.C. \& SAZIMA, M. 2003. The assemblage of flowers visited by hummingbirds in the "capões" of Southern Pantanal, Mato Grosso do Sul, Brazil. Flora 198: 427-435.

CAMARGO, G. 2003. Riqueza e diversidade de morcegos no Pantanal do Miranda-Abobral, Mato Grosso do Sul. Dissertação de mestrado, Universidade Federal de Mato Grosso do Sul, Campo Grande.

DAMASCENO, G.A., BEZERRA, M.A.O., BORTOLOTTO, I. \& POTT, A. 1999. Aspectos florísticos e fitofisionômicos dos capões do Pantanal do Abobral. In: Anais do II Simpósio sobre Recursos Naturais e Sócio-econômicos do Pantanal - Manejo e Conservação. CPAP Embrapa \& UFMS, Corumbá, p. 203-214.
EMMONS, L.H. \& FEER, F. 1990. Neotropical rain forest mammals. University of Chicago Press, Chicago.

FERNANDEZ, B.A., GUERRERO, R., LORD, R., OCHOA, J.G. \& ULLOA, G. 1988. Mamíferos de Venezuela: lista y claves para su identificación. Universidad Central de Venezuela, Faculdad de Agronomía, Maracay. 185 pp.

GENOWAYS, H.H., WILLIAMS, S.L. \& GROEN, J.A. 1981. Results of the Alcoa Foundation-Suriname Expedition. V. Noteworthy records of Surinamese mammals. Ann. Carnegie Mus. 50: 319-332.

JONES JR., J.K., ARROYO-CABRALES, J. \& OWEN, R.D. 1988. Revised checklist of bats (Chiroptera) of Mexico and Central America. Occas. Papers Mus., Texas Tech. Univ. 120: 1-34.

KOOPMAN, K.F. 1993. Order Chiroptera. Pp. 137-241 in Mammal Species of the World: a taxonomic and geographic reference (D. E. Wilson and D. M. Reeder, eds.). Smithsonian Institution Press. Washington.

MARINHO-FILHO, J. \& SAZIMA, I. 1998. Brazilian bats and conservation. In Bat Biology and Conservation ( $\mathrm{T}$. H. Kunz and P. Racey, eds.). Smithsonian Institution Press, p. 282-294.

MEDELLIN, R.A. 1983. Tonatia bidens and Mimon crenulatum in Chiapas, Mexico. J. Mammal. 64(1): 150.

OCHOA, J.G., MOLINA, C. \& GINER, S. 1993. Inventario y evaluación de las comunidades de mamíferos del Parque Nacional Canaima, con una lista de las especies registradas para la Guayana Venezolana. Acta Cient. Venezolana 44: 245-262.

ORTEGA, J., \& ARITA, H.T. 1997. Mammalian species: Mimon bennettii, 549: 1-4.

PEDRO, W.A., KOMENO, C.A.K. \& TADDEI, V.A. 1994. Morphometrics and biological notes on Mimon crenulatum (Chiroptera: Phyllostomidae). Bol. Mus. Para. Emilio Goeldi, ser. Zool. 10(1): 107-112.

PRANCE, G.T. \& SCHALLER, G. B. 1982. Preliminary study of some vegetation types of the Pantanal, Mato Grosso, Brazil. Brittonia 34: 228-251

Título: Primeiro registro do morcego Mimon crenulatum (Phyllostomidae) no Pantanal, sudoeste do Brasil

Autores: George Camargo \& Erich Fischer

Biota Neotropica, Vol. 5 ( número1): 2005

http://www.biotaneotropica.org.br/v5n1/pt/ abstract?short-communication+BN00705012005

Recebido: 23/08/2004

Revisado: $11 / 11 / 2004$

Publicado: 01/01/2005

ISSN 1676-0603 